

\title{
Somewhere Trivial Autohomeomorphisms
}

\author{
Saharon Shelah and Juris Steprāns
}

September 2, 2018

\begin{abstract}
It is show to be consistent that there is a non-trivial autohomeomorphism of $\beta \mathbb{N} \backslash \mathbb{N}$ while all such autohomeomorphisms are trivial on some open set. The model used is one due to Velickovic in which, coincidentally, Martin's Axiom also holds.
\end{abstract}

\section{Introduction}

An automorphism of of $\mathcal{P}(\omega) /[\omega]]^{<\aleph_{0}}$ - or, equivalently, an autohomeomorphism of $\beta \mathbb{N} \backslash \mathbb{N}$ - is said to be trivial if there is a bijection between cofinite subsets of $\omega$ which induces it; an automorphism is said to be somewhere trivial if its restriction to $\mathcal{P}(\mathcal{A})$ is trivial for some $A \in[\omega]^{\aleph_{0}}$. It was shown by Shelah, pages

*This research was completed while the first author was supported by the BSF and Rutgers University and the second author by NSERC and Rutgers University. This is publication Number 427 in the first author's list of publications. 
129 to 152 of [4], that it is equiconsistent with ZFC that all automorphisms of $\mathcal{P}(\omega) /[\omega]^{<\aleph_{0}}$ are trivial. The argument which proves this can be viewed as two distinct and almost independent arguments. The first part shows that it is consistent that every automorphism of $\mathcal{P}(\omega) /[\omega]^{<\aleph_{0}}$ is somewhere trivial while the second part expands on this argument to obtain the consistency of the assertion that all automorphisms are indeed trivial. Since the reasoning involved in both parts is, at least superficially, similar it is natural to ask whether it might not just be a consequence of the fact that every automorphism is somewhere trivial, that every automorphism is actually trivial. It is the purpose of this paper to show that such a theorem does not exist and hence, the second part of

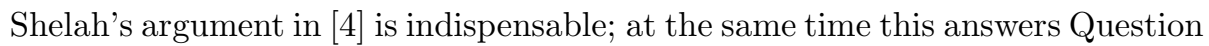
205 from [2].

In order to be more precise the following definitions will be introduced.

Definition 1.1 The relation $\equiv^{*}$ has the standard meaning - namely, $A \equiv^{*} B$ if and only if $|A \Delta B|<\aleph_{0}$ (here, $A \Delta B=(A \backslash B) \cup(B \backslash A)$ ). Also. $A \subseteq{ }^{*} B$ is defined to mean that $|B \backslash A|<\aleph_{0}$. If $A \subseteq \omega$ then the equivalence class of $A$ with respect to $\equiv^{*}$ will be denoted by $[A]$.

The notion of triviality can now be precisely formulated.

Definition 1.2 A homomorphism $\Phi: \mathcal{P}(\omega) /[\omega]^{<\aleph_{0}} \rightarrow \mathcal{P}(\omega) /[\omega]^{<\aleph_{0}}$ is said to be trivial on $A \subseteq \omega$ if there is $A^{\prime} \equiv^{*} A$ and a one-to-one function $f: A^{\prime} \rightarrow \omega$ such that $\Phi([B])=[f(B)]$ for every $B \subseteq A$. A homomorphism will be said 
to be somewhere trivial if there is some $A \in[\omega]^{\aleph_{0}}$ on which it is trivial. $A$ homomorphism is trivial if it is trivial on $\omega$.

It has already been mentioned that it was shown in [4] that it is consistent that all automorphisms of $\mathcal{P}(\omega) /[\omega]^{<\aleph_{0}}$ are trivial. The argument relied on the oracle chain condition and it was not clear what the effect of Martin's Axiom was on the question. This was partially answered in [5] where it was shown that PFA implies that all automorphisms of $\mathcal{P}(\omega) /[\omega]^{<\aleph_{0}}$ are trivial - for related results see [3]. The other half of the answer was provided by Velickovic in [8] where it is shown that it is consistent with Martin's Axiom that a nontrivial automorphism of $\mathcal{P}(\omega) /[\omega]<\aleph_{0}$ exists.

The following theorem of 8 offers an alternate characterisation of triviality which has proven to be very useful.

Lemma 1.1 (Velickovic) If $\Phi: \mathcal{P}(\omega) /[\omega]^{<\aleph_{0}} \rightarrow \mathcal{P}(\omega) /[\omega]^{<\aleph_{0}}$ is an automorphism and there exist Borel functions $\phi_{n}$ for $n \in \omega$ and a comeagre set $G \subset \mathcal{P}(\omega)$ such that for every $A \in G$ there is $n \in \omega$ such that $\left[\phi_{n}(A)\right]=\Phi([A])$ then $\Phi$ is trivial.

This is Theorem 2 of [8] except that in 8$]$ there is no reference to the comeagre set $G$; however an inspection of the proof in [8] will reveal that the hypothesis of Theorem 2 can be weakened to include $G$. Notice that if $\Phi: \mathcal{P}(\omega) /[\omega]<\aleph_{0} \rightarrow$ $\mathcal{P}(\omega) /[\omega]<\aleph_{0}$ is a trivial automorphism then it is simple to find a continuous function $\phi$ on $\mathcal{P}(\omega)$ such that $[\phi(A)]=\Phi([A])$ for each $A \subset \omega$. 
The notation $i_{X}$ will be used to denote the constant function whose domain is $X$ and which has value $i$ at each point in $X$. Whenever reference is made to a topology on $\mathcal{P}(\omega)$ this will be to the Cantor set topology under the canonical identification of $2^{\omega}$ with $\mathcal{P}(\omega)$ - in other words, a natural base for this topology consists of all sets of the form

$$
\left\{A \subseteq \omega: 1_{A} \cup 0_{\omega \backslash A} \supseteq g\right\}
$$

where $g$ is a finite partial function from $\omega$ to 2 .

The argument to be presented in the next section will be a modifiction and combination of arguments from pages 129 to 152 of [4], [5] and [8]. For the reader's benefit, some definitions and lemmas from [ [

Definition 1.3 An $\omega_{1}$-oracle is a sequence $\mathfrak{M}=\left\{\mathfrak{M}_{\xi}: \xi \in \omega_{1}\right\}$ such that

- $\mathfrak{M}_{\xi}$ is a countable, transitive model of $Z F C$ without the power set axiom

- $\xi \in \mathfrak{M}_{\xi}$ and $\mathfrak{M}_{\xi} \models \xi$ is countable

- $\left\{\xi \in \omega_{1}: A \cap \xi \in \mathfrak{M}_{\xi}\right\}$ contains a closed unbounded set for each $A \subseteq \omega_{1}$

Notice that the existence of an oracle requires that $\nabla_{\omega_{1}}$ is true.

Definition 1.4 If $\mathfrak{M}$ is an oracle then a partial order $\leq$ on $\omega_{1}$ (or some set coded by $\left.\omega_{1}\right)$ will be said to satisfy the $\mathfrak{M}$-chain condition if there is a closed unbounded set $C$ such that for every $\xi \in C$ and $A \subseteq \xi, A \in \mathfrak{M}_{\xi}$, if $A$ is predense in the order $(\xi, \leq \cap(\xi \times \xi))$ then it is predense in $\left(\omega_{1}, \leq\right)$. 
Further discussion of these definitions as well as proofs of the following lemmas can all be found in [4].

Lemma 1.2 Assume that $\diamond_{\omega_{1}}$ holds and $\phi_{\xi}(x)$ is a $\Pi_{2}^{1}$ formula - possibly with a real parameter - for each $\xi \in \omega_{1}$. Suppose also that there is no $r \in \mathbb{R}$ such that $\phi_{\xi}(r)$ holds for all $\xi \in \omega_{1}$ and that there is still no such $r$ even after adding a Cohen real. Then there is an oracle $\mathfrak{M}$ such that any partial order $\mathbb{Q}$ which satisfies the $\mathfrak{M}$-chain condition will not add $r \in \mathbb{R}$ such that $\phi_{\xi}(r)$ holds for all $\xi \in \omega_{1}$

Lemma 1.3 If $\left\{\mathfrak{M}^{\xi}: \xi \in \omega_{1}\right\}$ are oracles then there is a single oracle $\mathfrak{M}$ such that if any partial order satisfies the $\mathfrak{M}$-chain condition then it satisfies the $\mathfrak{M} \xi$ chain condition for each $\xi \in \omega_{1}$.

The oracle $\mathfrak{M}$ of Lemma 1.3 is easily decribed. It is the diagonal union of the oracles $\left\{\mathfrak{M}^{\xi}: \xi \in \omega_{1}\right\}$. This fact, rather than the statement of Lemma 1.3, will be used in the proof of Lemma 2.6.

Lemma 1.4 If $V$ is a model of $\diamond_{\omega_{1}}$ then there is, in $V$, an oracle $\mathfrak{M}$ such that if $\mathbb{Q}$ satisfies the $\mathfrak{M}$-chain condition then $1 \Vdash_{\mathbb{Q}}$ " $\mathbb{R} \cap V$ is second category"

Lemma 1.5 If $\mathfrak{M}$ is any oracle and $\mathbb{Q}$ satisfies the $\mathfrak{M}$-chain condition then $\mathbb{Q}$ satisfies the countable chain condition. 


\section{The proof}

The following partial order $\mathbb{P}$, was introduced by Velickovic in $[8]$ to add a nontrivial automorphism of $\mathcal{P}(\omega) /[\omega]^{<\aleph_{0}}$ while doing as little else as possible - at least assuming PFA.

Definition 2.1 The partial order $\mathbb{P}$ is defined to consist of all one-to-one functions $f: A \rightarrow B$ where

- $A \subseteq \omega$ and $B \subseteq \omega$

- for all $i \in \omega$ and $n \in \omega, f(i) \in\left(2^{n+1} \backslash 2^{n}\right)$ if and only if $i \in\left(2^{n+1} \backslash 2^{n}\right)$

- $\lim \sup _{n \rightarrow \omega}\left|\left(2^{n+1} \backslash 2^{n}\right) \backslash A\right|=\omega$ and hence, by the previous condition, $\lim \sup _{n \rightarrow \omega}\left|\left(2^{n+1} \backslash 2^{n}\right) \backslash B\right|=\omega$

The ordering on $\mathbb{P}$ is $\subseteq^{*}$.

The terms $2^{n}$ are not crucial since any sequence of intervals whose size tends to infinity could equally well have been used. Further modifications to the partial order are also possible — some can be found in [6] — but will not be important in the present context. It is however, useful to note the following.

Lemma 2.1 Assume $M A_{\lambda}$. Suppose that $\eta \leq \lambda$ and that

$$
\left\{f_{\xi}: \xi \in \eta\right\}
$$

is an increasing sequence from $\mathbb{P}$. Suppose further that there is $f^{\prime}$ such that $f^{\prime} \supseteq^{*} f_{\xi}$ for each $\xi \in \eta$. Then there is $f \in \mathbb{P}$ such that $f \supseteq^{*} f_{\xi}$ for each $\xi \in \eta$ 
Proof: It follows from $\mathrm{MA}_{\lambda}$ that there are $A$ and $B$ such that

- $A \supseteq^{*} \operatorname{dom}\left(f_{\xi}\right)$ for each $\xi \in \eta$

- $B \supseteq^{*} \operatorname{ran}\left(f_{\xi}\right)$ for each $\xi \in \eta$

- $\lim \sup _{n \rightarrow \omega}\left|\left(2^{n+1} \backslash 2^{n}\right) \backslash A\right|=\omega$

- $\lim \sup _{n \rightarrow \omega}\left|\left(2^{n+1} \backslash 2^{n}\right) \backslash B\right|=\omega$

Let $f=f^{\prime} \uparrow\left(A \cap\left(f^{\prime-1} B\right)\right)$.

Lemma 2.2 $\mathbb{P}$ is countably closed.

Proof: Given a sequence $\left\{f_{n}: n \in \omega\right\} \subseteq \mathbb{P}$ such that $f_{n} \subseteq^{*} f_{n+1}$ for each $n \in \omega$ choose inductively $k_{n}$ such that $f_{\omega}=\cup\left\{f_{n}\left\lceil\left(\omega \backslash k_{n}\right): n \in \omega\right\}\right.$ is a function. Now apply Lemma 2.1.

¿From Lemma 2.1 it follows that, given a sequence $\left\{f_{\xi}: \xi \in \omega_{1}\right\}$, it will be useful to find an element $f \in \mathbb{P}$ such that $f_{\xi} \subseteq^{*} f$ for each $\xi \in \omega_{1}$. The following partial order is designed to do precisely this.

Definition 2.2 Given $\left\{f_{\xi}: \xi \in \mu\right\}=\mathfrak{F}$ define $\mathbb{P}(\mathfrak{F})$ to be the partial order consisting of all $g \in \mathbb{P}$ such that there is some $\xi \in \mu$ such that $g \equiv^{*} f_{\xi}$. The ordering on $\mathbb{P}(\mathfrak{F})$ is $\subseteq$ as opposed to $\subseteq^{*}$ in $\mathbb{P}$. 
Definition 2.3 For any $G$ which is a centred subset of $\mathbb{P}$ define $\Phi_{G}: \mathcal{P}(\omega) /[\omega]<\aleph_{0} \rightarrow$ $\mathcal{P}(\omega) /[\omega]<\aleph^{<}$by

$$
\Phi_{G}([X])= \begin{cases}{[\{g(i): i \in X\}]} & \text { if }(\exists g \in G)(X \subseteq \operatorname{dom}(g)) \\ {[\omega \backslash\{g(i): i \in \omega \backslash X\}]} & \text { if }(\exists g \in G)(\omega \backslash X \subseteq \operatorname{dom}(g))\end{cases}
$$

If $\Phi$ is a $\mathbb{P}$-name for an automorphism of $\mathcal{P}(\omega) /[\omega]<\aleph_{0}$ then define $\partial_{G} \Phi(A)=B$ if and only if there is some $p \in G$ such that $p \vdash_{\mathbb{P}} " \Phi(A)=B$ ".

Velickovic showed that forcing with $\mathbb{P}$ yields a non-trivial automorphism of $\mathcal{P}(\omega) /[\omega]<\aleph_{0}$.

Theorem 2.1 If $G \subseteq \mathbb{P}$ is a generic filter on $\mathbb{P}$ then $\Phi_{G}$ is a non-trivial automorphism of $\mathcal{P}(\omega) /[\omega]^{<\aleph_{0}}$.

Proof: If it can be shown that $\operatorname{dom}\left(\Phi_{G}\right)=\mathcal{P}(\omega) /[\omega]^{<\aleph_{0}}=\operatorname{ran}\left(\Phi_{G}\right)$ then it is routine to check that $\Phi_{G}$ induces the desired autohomeomorphism of $\beta \mathbb{N} \backslash \mathbb{N}$. To see that this is so, assume that $p \in \mathbb{P}$ and $X \subseteq \omega-$ since $\mathbb{P}$ is countably closed, by Lemma 2.2, there is no harm in assuming that $X \in V$. It may also be assumed that $\lim \sup _{n \rightarrow \omega}\left|\left(2^{n+1} \backslash 2^{n}\right) \backslash(\operatorname{dom}(p) \cup X)\right|=\omega$ (otherwise deal with $\omega \backslash X)$. It must be shown that there is $p^{\prime} \supseteq p$ such that $p^{\prime} \Vdash_{\mathbb{P}}$ " $[X] \in \operatorname{dom}\left(\Phi_{G}\right)$ ". To do this let $p^{\prime} \supseteq p$ be any extension satisfying that $p^{\prime}(i) \in\left(2^{n+1} \backslash 2^{n}\right)$ if and only if $i \in\left(2^{n+1} \backslash 2^{n}\right)$ for all $i \in X$ and $n \in \omega$. A similar proof works for the range of $\Phi$.

An important fact is the result of Velickovic [8] that if $F$ is $\mathbb{P}$-generic over a model $V$ of PFA, 
then in $V[F]$, not only is there is a non-trivial autohomeomorphism of $\beta \mathbb{N} \backslash \mathbb{N}$, but MA also holds. It will be shown that a closer analysis of this model yields that in $V[F]$ all autohomeomorphisms of $\beta \mathbb{N} \backslash \mathbb{N}$ are somewhere trivial.

Loosely speaking, the following theorem will show that if $\Phi: \mathcal{P}(\omega) /[\omega]<\aleph_{0} \rightarrow$ $\mathcal{P}(\omega) /[\omega]<\aleph_{0}$ is a nontrivial automorphism then it is still nontrivial after adding a Cohen real.

Lemma 2.3 If $\Phi \in V$ is not trivial and $V^{\prime}$ is obtained by adding a Cohen real to $V$ then, in $V^{\prime}$, there do not exist Borel functions $\left\{\psi_{n}: n \in \omega\right\}$ such that for each $C \in \mathcal{P}(\omega) \cap \mathcal{V}$ there is some $n \in \omega$ such that $\Phi([C])=\left[\psi_{n}(C)\right]$.

Proof: Suppose that $V^{\prime}$ is obtained by forcing with the countable partial order $\mathbb{C}$ and that $\psi_{n}$ are $\mathbb{C}$-names for Borel functions such that for each $C \in \mathcal{P}(\omega) \cap \mathcal{V}$ there is some $n \in \omega$ such that $\Phi([C])=\left[\psi_{n}(C)\right]$. Let $G_{n}$ be a name for a comeagre set such that $\psi_{n}\left\lceil G_{n}\right.$ is continuous. Define $\psi_{n}^{p}=\left\{(A, B): p \Vdash_{\mathbb{C}}\right.$ " $\psi_{n}(A)=B$ and $A \in G_{n}$ " $\}$. Let $D_{n}^{p}$ be the closure of the domain of $\psi_{n}^{p}$ and let $E_{n}^{p}$ be the closure of the interior of $D_{n}^{p}$ - note that $D_{n}^{p} \backslash E_{n}^{p}$ is meagre. Let $f_{n}^{p}$ be the maximal extension of $\psi_{n}^{p}$ to a continuous function on $E_{n}^{p}$.

It must be that case that the domain of $f_{n}^{p}$ is comeagre in $E_{n}^{p}$ because if the domain of $f_{n}^{p}$ is not comeagre in $E_{n}^{p}$ then, because it is Borel, there must be some open set $U \subset E_{n}^{p}$ such that the set of points in $U$ to which $\psi_{n}^{p}$ can be continuously extended is meagre in $U$. Since $p \Vdash$ " $\psi_{n}^{p} \subset \psi_{n}$ " and because being a meagre Borel set absolute, it must be that the set of points in $U$ to which $\psi_{n}$ can be continuously extended is also meagre in $U$. The reason is that the 
domain $\psi_{n}^{p}$ is dense in $E_{n}^{p}$ and so it follows that the domain $\psi_{n}^{p}$ is dense in $U$ and, moreover, not being a point to which a function can be continuously extended is an absolute property. This contradicts the fact that $G_{n}$ is comeagre.

Now let $M^{\prime}=\cup\left\{D_{n}^{p} \backslash E_{n}^{p}: n \in \omega\right.$ and $\left.p \in \mathbb{C}\right\} \cup\left\{E_{n}^{p} \backslash \operatorname{dom}\left(f_{n}^{p}\right): n \in \omega\right\}$ and observe that $M^{\prime}$ is meagre. Now recall the following fact: If $V$ is a model of $Z F C$ and $r$ is a Cohen real and $N \in V[r]$ is a meagre set then there is a meagre set $N^{\prime} \in V$ such that $N \cap V \subset N^{\prime} \cap V$. Let $N$ be a meagre set such that $G_{n} \supset \mathcal{P}(\omega) \backslash N$ for each $n \in \omega$. Let $M=M^{\prime} \cup N$. It is true in $V^{\prime}$ that for every $A \in(\mathcal{P}(\omega) \backslash M) \cap V$ there is some $p \in \mathbb{C}$ such that $\left[\psi_{n}^{p}(A)\right]=\Phi([A])$. Since this statement is arithmetic in the parameters $A$ and $\Phi([A])$ - and both of these parameters belong to $V$ - this must be true in $V$ also. Now apply Lemma 1.1 .

Lemma 2.4 Given $\eta \in \omega_{1}$, a sequence $\left\{f_{\xi}: \xi \in \eta\right\}=\mathfrak{F}_{\eta}$ and a countable elementary submodel $\mathfrak{A} \prec\left(\mathfrak{H}\left(\omega_{2}\right), \in\right)$, such that $\mathfrak{F}_{\eta} \in \mathfrak{A}$, there is $f \in \mathbb{P}$ which is A-generic for $\mathbb{P}\left(\mathfrak{F}_{\eta}\right)$. Moreover, for any extension $\left\{f_{\xi}: \xi \in \mu\right\}=\mathfrak{F}_{\mu}$ of $\mathfrak{F}_{\eta}$ such that $\eta \in \mu \in \omega_{1}$ and $f_{\eta}=f$, every $D \in \mathfrak{A}$ is predense in $\mathbb{P}\left(\mathfrak{F}_{\mu}\right)$ provided that it is dense in $\mathbb{P}\left(\mathfrak{F}_{\eta}\right)$.

Proof: Let $\left\{E_{k}: k \in \omega\right\}$ enumerate all dense subsets of $\mathbb{P}\left(\mathfrak{F}_{\eta}\right)$ in $\mathfrak{A}$. Construct sequences $\left\{g_{n}: n \in \omega\right\}$ and $\left\{K_{n}: n \in \omega\right\}$ such that for all $n \in \omega$

- $g_{n} \subseteq g_{n+1}$ 
- $K_{n}<K_{n+1}$

- $g_{n+1}\left\lceil K_{n}=g_{n} \uparrow K_{n}\right.$

- there is some $i$ such that $2^{i+1}=K_{n}$ and $\left|\left(2^{i+1} \backslash 2^{i}\right) \backslash \operatorname{dom}\left(g_{n}\right)\right| \geq n$

- for each bijection $t: K_{n} \rightarrow K_{n}$ there $h \in \cap_{j \in n+1} E_{j}$ such that $t \cup g_{n} \uparrow$ $\left(\omega \backslash K_{n}\right) \supseteq h$

It is easy to see that this can be done. Hence, it is possible to define $f=\cup\left\{g_{n}\right.$ : $n \in \omega\}$. Notice that $\left\{g: g \supseteq^{*} f_{\xi}\right\}$ is dense in $\mathbb{P}\left(\mathfrak{F}_{\eta}\right)$ and definable in $\mathfrak{A}$ - hence $f \supseteq^{*} f_{\xi}$ for each $\xi \in \eta$. To check that $f$ has the desired properties suppose that $g \in \mathbb{P}\left(\mathfrak{F}_{\mu}\right)$ for some $\mu \geq \eta$ and that $f_{\eta}=f$. If $E$ is dense in $\mathbb{P}\left(\mathfrak{F}_{\eta}\right)$ then there is some $m$ such that $E \in\left\{E_{j}: j \in m+1\right\}$ and $g\left\lceil\left(\omega \backslash K_{m}\right) \supseteq f \uparrow\left(\omega \backslash K_{m}\right)\right.$. By extending $g$ if necessary, it may, without loss of generality, be assumed that $g \uparrow K_{m}=t$ and that $t: K_{m} \rightarrow K_{m}$ is a bijection. It follows that $t \cup g_{m} \uparrow$ $\left(\omega \backslash K_{m}\right) \supseteq h$ for some $h \in E$ and hence $g \supseteq h \in E$.

Lemma 2.5 Suppose that $V$ is a model of $2^{\aleph_{0}}=\aleph_{1}$. If $\Phi$ is a $\mathbb{P}$-name for a nowhere trivial automorphism of $\mathcal{P}(\omega) /[\omega]^{<\aleph_{0}}$ and $f \in \mathbb{P}$ then there is a sequence $\mathfrak{F}=\left\{\mathfrak{f}_{\xi}: \xi \in \omega_{1}\right\} \subset \mathbb{P}$ such that $f_{0}=f$ and $\partial_{\mathfrak{F}} \Phi$ is nowhere trivial.

Proof: Let $\left\{A_{\xi}: \xi \in \omega_{1}\right\}$ be an enumeration of $\mathcal{P}(\omega) /[\omega]<\aleph_{0}$ in $V$ and

$$
\left\{\Psi_{\xi}: \mathcal{P}\left(\mathcal{C}_{\xi}\right) \rightarrow \mathcal{P}\left(\mathcal{B}_{\xi}\right): \xi \in \omega_{\infty}\right\}
$$


enumerate all possible names for continuous functions from a Borel comeagre subset of some $\mathcal{P}(\mathcal{C})$ to some $\mathcal{P}(\mathcal{B})$ so that each name occurs cofinally often. It suffices to construct $\mathfrak{F}=\left\{\mathfrak{f}_{\xi}: \xi \in \omega_{1}\right\} \subseteq \mathbb{P}$ by induction so that for every limit ordinal $\xi$ the following conditions are satisfied

- $f_{\xi+n}$ decides, in $\mathbb{P}$, the values of $\Phi\left(A_{\xi+n-2}\right)$ and $\Phi^{-1}\left(A_{\xi+n-2}\right)$ for $n \geq 2$

- there is some $C \subset A_{\xi}$ such that $1 \Vdash_{\mathbb{P}(\mathfrak{F})}$ " $\partial_{\mathfrak{F}} \Phi(C) \neq\left[\Psi_{\xi}(C)\right]$ "

It is possible to construct $\mathfrak{F}$ inductively because a failure would mean that for some $\xi \in \omega_{1}$ it must be the case that

$$
f_{\xi} \Vdash_{\mathbb{P}} " \Phi \text { is trivial on } A_{\xi} "
$$

contradicting that $\Phi$ is a name for a nowhere trivial automorphism of $\mathcal{P}(\omega) /[\omega]<\aleph_{0}$.

Lemma 2.6 Suppose that $V$ is a model of $\diamond$ and that $\Phi$ is a $\mathbb{P}$-name for a nowhere trivial automorphism of $\mathcal{P}(\omega) /[\omega]^{<\aleph_{0}}$. Then there is a sequence $\mathfrak{F}=$ $\left\{\mathfrak{f}_{\xi}: \xi \in \omega_{1}\right\} \subset \mathbb{P}$ such that

- $\mathbb{P}(\mathfrak{F})$ satisfies the countable chain condition

- $\mathbb{R} \cap V$ is of second category after forcing with $\mathbb{P}(\mathfrak{F})$

- for every $G \subset \mathbb{P}(\mathfrak{F})$ which is generic over $V$, for every $A \in V \cap \mathcal{P}(\omega)$, $B \in V \cap \mathcal{P}(\omega)$ and $\mathbb{P}(\mathfrak{F})$-names $\Psi_{n}$ such that for each $n \in \omega$

$1 \Vdash_{\mathbb{P}(\mathfrak{F})} " \Psi_{n}: \mathcal{P}(\mathcal{A}) \rightarrow \mathcal{P}(\mathcal{B})$ is continuous" 
there is some $C \in V$ such that $\partial_{\mathfrak{F}} \Phi([C]) \neq \Psi_{n}([C])$ for all $n \in \omega$

In the last clause the possibility that $C \notin \operatorname{dom}\left(\Psi_{n}\right)$ is allowed in the sense that if $C \notin \operatorname{dom}\left(\Psi_{n}\right)$ then $\partial_{\mathfrak{F}} \Phi([C]) \neq \Psi_{n}([C])$.

Proof: The proof will be rely on constructing a particular oracle which will guarantee that the three clauses are all satisfied. The only wrinkle is that the oracle and the sequence $\left\{f_{\xi}: \xi \in \omega_{1}\right\}$ must be constructed simultaneously. The sequence $\left\{f_{\xi}: \xi \in \omega_{1}\right\}$ will be obtained by diagonalizing across $\aleph_{1}$ such sequences.

In particular, let $\mathfrak{N}$ be any oracle such that forcing with an $\mathfrak{N}$-oracle chain condition partial order preserves the fact that $\mathbb{R} \cap V$ is of second category such an oracle exists by Lemma 1.4. Then construct sequences $\mathfrak{F}^{\mu}=\left\{\mathfrak{f}_{\xi}^{\mu}: \xi \in\right.$ $\left.\omega_{1}\right\} \subset \mathbb{P}$ and $\mathfrak{M}^{\mu}=\left\{\mathfrak{M}_{\xi}^{\mu}: \xi \in \omega_{1}\right\} \subset \mathbb{P}$ for $\mu \in \omega_{1}$ such that

a. $f_{\mu}^{\xi}=f_{\mu}^{\mu}$ if $\mu<\xi$

b. $\partial_{\mathfrak{F}^{\mu}} \Phi$ is nowhere trivial for $\mu \in \omega_{1}$

c. for $\mu \in \omega_{1}$, if $\mathbb{Q}$ satisfies the $\mathfrak{M}^{\mu}$-chain condition and $G$ is $\mathbb{Q}$-generic over $V$ then, in $V[G]$, for every $A \in V \cap \mathcal{P}(\omega), B \in V \cap \mathcal{P}(\omega)$ there do not exist $\left\{\Psi_{n}: n \in \omega\right\}$ such that $\Psi_{n}: \mathcal{P}(\mathcal{A}) \rightarrow \mathcal{P}(\mathcal{B})$ is continuous and for all $C \in \mathcal{P}(\mathcal{A}) \cap \mathcal{V}$ there exists $n \in \omega$ such that $\partial_{\mathfrak{F}^{\mu}} \Phi([C])=\Psi_{n}([C])$

d. $\left\{f_{\mu}^{\xi}:\{\mu, \xi\} \in[\eta]^{2}\right\} \in \mathfrak{M}_{\eta}^{\eta}$ for each $\eta \in \omega_{1}$

e. $f_{\mu}^{\mu+1}$ is $\mathbb{P}\left(\left\{f_{\xi}^{\xi}: \xi \in \mu\right\}\right)$-generic over $\mathfrak{M}_{\mu+1}^{\mu}$ 
f. $\mathfrak{M}_{\xi}^{\mu} \in \mathfrak{M}_{\xi}^{\eta}$ if $\xi \in \mu \in \eta$

g. $\mathfrak{M}^{\mathfrak{O}}=\mathfrak{N}$

To see that this suffices let $\mathfrak{M}_{\xi}=\mathfrak{M}_{\xi+1}^{\xi}$ and let $\mathfrak{F}=\left\{\mathfrak{f}_{\xi}=\mathfrak{f}_{\xi+1}^{\xi+1}: \xi \in\right.$ $\left.\omega_{1}\right\}$. It follows from the remark following Lemma 1.3 that $\left\{\mathfrak{M}_{\xi}: \xi \in \omega_{1}\right\}$ is an oracle and that any partial order which satisfies the $\left\{\mathfrak{M}_{\xi}: \xi \in \omega_{1}\right\}$-chain condition also satisfies each of the $\mathfrak{M}^{\mu}$-chain conditions for $\mu \in \omega_{1}$. Since $f_{\mu}^{\mu+1}$ is $\mathbb{P}\left(\left\{f_{\xi}^{\xi}: \xi \in \mu\right\}\right)$-generic over $\mathfrak{M}_{\mu+1}^{\mu}$ it follows that $\mathbb{P}\left(\left\{f_{\xi}: \xi \in \omega_{1}\right\}\right)$ satisfies the $\left\{\mathfrak{M}_{\xi}: \xi \in \omega_{1}\right\}$ chain condition. In particular, this partial order satisfies the $\mathfrak{M}^{\circ}$-chain condition and hence the second clause of the theorem will be satisfied. That the first clause is satisfied follows from Lemma 1.5. So it only remains to be shown that the last clause is satisfied.

To this end, suppose that $A \in V \cap \mathcal{P}(\omega), B \in V \cap \mathcal{P}(\omega)$ and $\mathbb{P}(\mathfrak{F})$-names $\Psi_{n}$ are given such that for each $n \in \omega$

$$
1 \Vdash_{\mathbb{P}(\mathfrak{F})} " \Psi_{n}: \mathcal{P}(\mathcal{A}) \rightarrow \mathcal{P}(\mathcal{B}) \text { is continuous" }
$$

Since $\mathbb{P}(\mathfrak{F})$ satisfies the countable chain condition, there is some $\gamma \in \omega_{1}$ such that $\mathfrak{M}_{\gamma}$ models that for each $n \in \omega$

$$
1 \Vdash_{\mathbb{P}\left(\left\{f_{\xi}: \xi \in \gamma\right\}\right)} " \Psi_{n}: \mathcal{P}(\mathcal{A}) \rightarrow \mathcal{P}(\mathcal{B}) \text { is continuous" }
$$

It now follows that this statement about $\mathfrak{M}_{\gamma}=\mathfrak{M}_{\gamma}^{\gamma}$ must be true in $\mathfrak{M}_{\gamma+1}^{\gamma}$ because $\mathfrak{M}_{\gamma}^{\gamma} \in \mathfrak{M}_{\gamma+1}^{\gamma}$. But it now follows from the fact that $f_{\gamma}^{\gamma+1}$ is generic over 
$\mathfrak{M}_{\gamma+1}^{\gamma}$ that

$$
f_{\gamma}^{\gamma+1} \Vdash_{\mathbb{P}\left(\left\{f_{\xi}^{\xi} ; \xi \in \gamma\right\}\right)} "(\exists C \in \mathcal{P}(\mathcal{A}) \cap \mathcal{V})(\forall \backslash \in \omega) \partial_{\mathfrak{F}^{\gamma}} \oplus([\mathcal{C}]) \neq \ominus_{\backslash}([\mathcal{C}]) "
$$

Since $f_{\gamma}^{\gamma+1} \supset^{*} f_{\mu}^{\gamma+1}=f_{\mu}^{\mu}$ for each $\mu \in \omega_{1} \cap \mathfrak{M}_{\gamma+1}^{\gamma}$ it follows that $\partial_{\mathfrak{F}} \Phi\left\lceil\mathfrak{M}_{\gamma+1}^{\gamma}=\right.$ $\partial_{\mathfrak{F}^{\gamma}}\left\lceil\mathfrak{M}_{\gamma+1}\right.$ and hence

$$
f_{\gamma}^{\gamma+1} \Vdash_{\mathbb{P}\left(\left\{f_{\xi}^{\xi} ; \xi \in \gamma\right\}\right)} "(\exists C \in \mathcal{P}(\mathcal{A}) \cap \mathcal{V})(\forall \backslash \in \omega) \partial_{\mathfrak{F}} \oplus([\mathcal{C}]) \neq \ominus_{\backslash}([\mathcal{C}]) "
$$

Since the necessary dense sets are definable in $\mathfrak{M}_{\gamma+1}^{\gamma}=\mathfrak{M}_{\gamma}$ it follows that

$$
f_{\gamma} \Vdash_{\mathbb{P}(\mathfrak{F})} "(\exists C \in \mathcal{P}(\mathcal{A}) \cap \mathcal{V})(\forall \backslash \in \omega) \partial_{\mathfrak{F}} \oplus([\mathcal{C}]) \neq \ominus_{\backslash}([\mathcal{C}]) "
$$

which is what is required.

All that remains to be done is to show that the inductive construction can be completed. For this, suppose that $\mathfrak{F}^{\mu}=\left\{\mathfrak{f}_{\xi}^{\mu}: \xi \in \omega_{1}\right\}$ and $\mathfrak{M}^{\mu}=\left\{\mathfrak{M}_{\xi}^{\mu}: \xi \in \omega_{1}\right\}$ have been constructed for $\mu \in \eta$.

If $\eta$ is a limit then it is easy to use Lemma 2.5 in order to satisfy conditions (a) and (b). If $\eta$ is a successor then Lemma 2.4 must also be used in order to satisfy condition (e). To construct $\mathfrak{M}_{\xi}^{\eta}$ for $\xi \in \omega_{1}$ use Lemmas 1.2 and Lemma 2.3 to satisfy condition (c). It is then easy to enlarge the terms of the oracle to satisfy conditions (d) and (f).

The proof of the main theorem will require the following definition, which is a reformulated form of the partial order which appeared in [4] on page 134.

Definition 2.4 Given a sequence $\left\{\left(W_{\xi}, V_{\xi}\right): \xi \in \eta\right\}$ define $\mathbb{Q}\left(\left\{\left(W_{\xi}, V_{\xi}\right): \xi \in\right.\right.$ $\eta\})$ to be the partial order which consists of all functions $g$ such that there is 
$\Gamma \in[\eta]^{<\aleph_{0}}$ such that

$$
g \equiv^{*} \cup\left\{0_{V_{\xi}} \cup 1_{W_{\xi} \backslash V_{\xi}}: \xi \in \Gamma\right\}
$$

The ordering on $\mathbb{Q}\left(\left\{\left(W_{\xi}, V_{\xi}\right): \xi \in \eta\right\}\right)$ is inclusion.

Theorem 2.2 It is consistent, relative to the consistency of ZFC and PFA, that all automorphisms of $\mathcal{P}(\omega) /[\omega]^{<\aleph_{0}}$ are somewhere trivial but there is, nevertheless, a non-trivial automorphism of $\mathcal{P}(\omega) /[\omega]]^{<\aleph_{0}}$.

Proof: It follows from Theorem 2.1 that forcing with $\mathbb{P}$ yields a non-trivial automorphism of $\mathcal{P}(\omega) /[\omega]^{<\aleph_{0}}$. Hence all that needs to be shown is that in the resulting model all automorphisms of $\mathcal{P}(\omega) /[\omega]^{<\aleph_{0}}$ are somewhere trivial. To do this suppose that $V$ is a model of PFA and that in this model

$$
\left.1 \Vdash_{\mathbb{P}} \text { " } \Phi \text { is a nowhere trivial automorphism of } \mathcal{P}(\omega) /[\omega]\right]^{<\aleph_{0}} \text {. }
$$

Let $G$ be a $V$-generic filter on a countably closed partial order - the Levy collapse of $2^{\aleph_{0}}$ to $\aleph_{1}$ for example — which forces the existence of a $\diamond$-sequence. Let $\mathfrak{F}=\left\{\mathfrak{f}_{\xi}: \xi \in \omega_{1}\right\}$ be some fixed sequence with the properties guaranteed by Lemma 2.6 In particular, Lemma 2.6 guarantees that for every $A \in V[G] \cap \mathcal{P}(\omega)$, $B \in V[G] \cap \mathcal{P}(\omega)$ and for every collection of $\mathbb{P}(\mathfrak{F})$-names $\left\{\Psi_{n}: n \in \omega\right\}$ such that, for each $n \in \omega$,

$$
1 \Vdash_{\mathbb{P}(\mathfrak{F})} \text { " } \Psi_{n}: \mathcal{P}(\mathcal{A}) \rightarrow \mathcal{P}(\mathcal{B}) \text { is continuous" }
$$

there is some $C \in \mathcal{P}(\mathcal{A}) \cap \mathcal{V}[\mathcal{G}]=\mathcal{P}(\mathcal{A}) \cap \mathcal{V}$ such that $\partial_{\mathfrak{F}} \Phi([C]) \neq \Psi_{n}([C])$ for every $n \in \omega$. Let $H$ be $V[G]$-generic for the partial order $\mathbb{P}(\mathfrak{F})$. 
Let $\mathfrak{M}$ be an arbitrary oracle in $V$. A sequence $\left\{\left(W_{\xi}, V_{\xi}\right): \xi \in \omega_{1}\right\}$ will be constructed in $V[G][H]$ so that

- if $\mathbb{Q}_{\xi}=\mathbb{Q}\left(\left\{\left(W_{\eta}, V_{\eta}\right): \eta \in \xi\right\}\right)$ then $\mathbb{Q}_{\omega_{1}}$ satisfies the $\mathfrak{M}$-chain condition

- $\left(W_{\xi}, V_{\xi}\right) \in V[G]=V \cap(\mathcal{P}(\omega))^{2}$

- $V_{\xi} \subseteq W_{\xi} \subseteq \omega$

- $\left|W_{\xi} \cap W_{\eta}\right|<\aleph_{0}$ if $\eta \neq \xi$

- for each $p \in \mathbb{Q}_{\xi}$ and $\mathbb{Q}_{\xi}$-name, $Y \in \mathfrak{M}_{\xi}$, for a subset of $\omega$

$$
p \cup 1_{V_{\xi}} \cup 0_{W_{\xi} \backslash V_{\xi}} \Vdash_{\mathbb{Q}_{\xi+1}} " \partial_{\mathfrak{F}} \Phi\left(\left[W_{\xi}\right]\right) \cap[Y] \neq \partial_{\mathfrak{F}} \Phi\left(\left[V_{\xi}\right]\right) "
$$

- the dense subsets of $\mathbb{Q}_{\xi+1}$ which guarantee that the previous statement is true are predense in $\mathbb{Q}_{\omega_{1}}$

Before continuing, define $\Phi^{*}(A) \subseteq \omega$ arbitrarily to satisfy that $\left[\Phi^{*}(A)\right]=$ $\partial_{\mathfrak{F}} \Phi([A])$ for each $[A] \in \operatorname{dom}\left(\partial_{\mathfrak{F}} \Phi\right)$. Next, choose an almost disjoint family $\left\{W_{\xi}^{\prime}: \xi \in \omega_{1}\right\}$ in the model $V[G]$. The set $W_{\xi}$ will be chosen so that, among other things, $W_{\xi} \subseteq W_{\xi}^{\prime}$ - this will, of course, guarantee that the resulting family is almost disjoint. If this construction succeeds then it is possible to proceed as in $\left[5\right.$ to prove that forcing with $\mathbb{P}(\mathfrak{F}) * \mathbb{Q}_{\omega_{1}}$ adds a set to which the partial automorphism $\partial_{\mathfrak{F}} \Phi$ can not be extended.

In particular, if $H_{1} * H_{2}$ is $\mathbb{P}(\mathfrak{F}) * \mathbb{Q}_{\omega_{1}}$ generic then, setting $X=\cup\left\{f^{-1}(\{1\})\right.$ : $\left.f \in H_{2}\right\}$, it follows that $X \cap W_{\xi} \equiv^{*} V_{\xi}$ for each $\xi \in \omega_{1}$ but, in $V[G]\left[H_{1} * H_{2}\right]$, for 
every $Y \subseteq \omega$ there is $\beta \in \omega_{1}$ such that $\Phi^{*}\left(W_{\xi}\right) \cap Y \not \equiv^{*} \Phi^{*}\left(V_{\xi}\right)$ for each $\xi \geq \beta$. Just as in [5], it is possible to define a relation $R$ on $\omega_{1}$ by $R(\xi, \eta)$ holds if and only if either $\left(\Phi^{*}\left(W_{\xi}\right) \backslash \Phi^{*}\left(V_{\xi}\right)\right) \cap \Phi^{*}\left(V_{\eta}\right) \neq \emptyset$ or $\left(\Phi^{*}\left(W_{\eta}\right) \backslash \Phi^{*}\left(V_{\eta}\right) \cap \Phi^{*}\left(V_{\xi}\right)\right) \neq \emptyset$. It is easy to see that this is a semiopen relation — as defined in [1] - and that moreover, there is no $S \in\left[\omega_{1}\right]^{\aleph_{1}}$ such that $[S]^{2} \cap R=\emptyset$. The reason for the last statement is that otherwise, letting $Y=\cup\left\{\Phi^{*}\left(V_{\xi}\right): \xi \in S\right\}$ would yield a contradiction to the fact that $\Phi^{*}\left(W_{\xi}\right) \cap Y \not^{*} \Phi^{*}\left(V_{\xi}\right)$ for all but countably many $\xi$. Hence, by the results of [1], there is a proper partial order $\mathbb{K}$ which adds a set $S \in\left[\omega_{1}\right]^{\aleph_{1}}$ such that $[S]^{2} \subseteq R$. This makes the fact that $\partial_{\mathfrak{F}} \Phi$ can not be extended to the set $X$ absolute. The reason for this is that if there is a set $Y$ such that $\partial_{\mathfrak{F}} \Phi([X])$ can be defined to be $[Y]$, then it must be the case that $Y \cap \Phi^{*}\left(W_{\xi}\right) \equiv^{*} \Phi^{*}\left(V_{\xi}\right)$ for each $\xi \in S$. But then there is an uncountable set $S^{\prime} \subseteq S$, as well as $J \in \omega$, such that $Y \cap \Phi^{*}\left(W_{\xi}\right) \backslash J=\Phi^{*}\left(V_{\xi}\right) \backslash J$ for each $\xi \in S^{\prime}$. It follows that $\Phi^{*}\left(V_{\xi}\right) \backslash J \subseteq Y$ and that $\left(\Phi^{*}\left(W_{\xi}\right) \backslash \Phi^{*}\left(V_{\xi}\right)\right) \backslash J \subseteq \omega \backslash Y$ for each $\xi \in S^{\prime}$. Choosing $\xi$ and $\zeta$ in $S^{\prime}$ such that $\Phi^{*}\left(V_{\xi}\right) \cap J=\Phi^{*}\left(V_{\zeta}\right) \cap J$ and $\Phi^{*}\left(W_{\xi}\right) \cap J=\Phi^{*}\left(W_{\zeta}\right) \cap J$ yields the desired contradiciton.

The iteration $\mathbb{D} * \mathbb{P}(\mathfrak{F}) * \mathbb{Q} \omega_{1} * \mathbb{K}$ is proper and only $\aleph_{1}$ dense sets in it need be met in order to obtain $S$ and the set $X$ such that $\partial_{\mathfrak{F}} \Phi$ can not be extended to include $[X]$ in its domain. Let $f_{\omega_{1}}$ be the element of $\mathbb{P}$ obtained by forcing with $\mathbb{P}\left(\{f r a k F)\right.$ and Lemma 2.1 and note that, in $V, f_{\omega_{1}} \Vdash_{\mathbb{P}} " \Phi$ does not extend to $X "$ because $f_{\omega_{1}} \Vdash_{\mathbb{P}}$ " $\partial_{\mathfrak{F}} \Phi \subseteq \Phi "$ 
Hence it may be assumed that the construction breaks down at some point $\mu \in \omega_{1}$. What can go wrong? First, there are certain predense sets required at stage $\mu$ which must remain predense in the partial order $\mathbb{Q}_{\mu+1}$. It is shown on page 134 of 4 that, for each predense set $E \subseteq \mathbb{Q}_{\mu}$, there is a dense open set $\mathcal{W} \subseteq \mathcal{P}\left(\mathcal{W}_{\mu}^{\prime}\right)$ such that if $W \in \mathcal{W}$ then, letting $W_{\mu}=W, E$ remains predense in $\mathbb{Q}_{\mu+1}$ for any $V=V_{\mu} \subseteq W_{\mu}$. Note that $\mathcal{W}$ is closed under the operation of taking infinite subsets. Recall that $\mathfrak{F}$ was chosen so that $\mathcal{P}\left(\mathcal{W}_{\mu}^{\prime}\right) \cap \mathcal{V}[\mathcal{G}]$ is of second category in $\mathcal{P}\left(\mathcal{W}_{\mu}^{\prime}\right)$ in the model $V[G]\left[H_{1}\right]$. It follows that it may be assumed that $W_{\mu} \in V[G]$ and that $W_{\mu} \in \mathcal{O}$ for every open set $\mathcal{O} \subseteq \mathcal{P}\left(\mathcal{W}_{\mu}^{\prime}\right)$ which is definable from $\mathfrak{M}_{\mu}$ and $\left\{\left(W_{\xi}, V_{\xi}\right): \xi \in \mu\right\}$ - but note that $\mathbb{Q}_{\mu}$ is definable from $\left\{\left(W_{\xi}, V_{\xi}\right): \xi \in \mu\right\}$. Hence the only possible problem is that it is not possible to find $V_{\mu} \subseteq W_{\mu}$ satisfying the required properties - namely, there is no $V_{\mu} \subseteq W_{\mu}$ such that for each $p \in \mathbb{Q}_{\mu}$ and $\mathbb{Q}_{\mu}$-name, $Y \in \mathfrak{M}_{\mu}$, for a subset of $\omega$

$$
p \cup 1_{V_{\mu}} \cup 0_{W_{\mu} \backslash V_{\mu}} \Vdash_{\mathbb{Q}_{\mu+1}} " \partial_{\mathfrak{F}} \Phi\left(\left[W_{\mu}\right]\right) \cap[Y] \not \#^{*} \partial_{\mathfrak{F}} \Phi\left(\left[V_{\mu}\right]\right) " .
$$

To see that this can not happen it will be necessary to discuss forcing in $\mathbb{Q}_{\mu+1}$ before $\mathbb{Q}_{\mu+1}$ has been defined - namely, before $V_{\mu}$ has been defined. This will be done by defining $\Vdash_{*}$ as follows: If $p \in \mathbb{Q}_{\mu}, X$ and $Y$ are $\mathbb{Q}_{\mu}$ names for subsets of $\omega$ and $f: W_{\mu} \rightarrow 2$ is a partial function, define

$$
(p, f) \Vdash_{*} " X \not \equiv^{*} Y "
$$


if and only if for each $p^{\prime} \in \mathbb{Q}_{\mu}$ such that $p^{\prime} \cup p \cup f$ is a function and for each $n \in \omega$ there is $p^{\prime \prime}$ such that

- $p^{\prime \prime} \cup p^{\prime} \cup p \cup f$ is a function

- $p^{\prime \prime} \Vdash_{\mathbb{Q}_{\mu}} " k \in X \Delta Y$ " for some $k \geq n$

Claim 1 If the following conditions are satisfied

- $(p, f) \Vdash_{*} " X^{\prime} \cap X \not \equiv * Y "$

- $X^{\prime}$ is a $\mathbb{Q}_{\mu}$ name belonging to $\mathfrak{M}_{\mu}$

- $X$ and $Y$ are subsets of $\omega$ in $V[G]$

- $V_{\mu}=f^{-1}(\{1\})$

then then $p \cup f \Vdash_{\mathbb{Q}_{\mu+1}} " X \cap X^{\prime} \not \equiv^{*} Y "$.

The way to see this is to note that the following statements are all equivalent

- $p^{\prime \prime} \Vdash_{\mathbb{Q}_{\mu}} " k \in X \cap X^{\prime} \Delta Y "$

- $k \in X \backslash Y$ and $p^{\prime \prime} \Vdash_{\mathbb{Q}_{\mu}}$ " $k \in X^{\prime \prime}$ or $k \in Y \backslash X$ and $p^{\prime \prime} \Vdash_{\mathbb{Q}_{\mu}} " k \notin X^{\prime} "$

- $k \in X \backslash Y$ and $p^{\prime \prime} \Vdash_{\mathbb{Q}_{\mu+1}} " k \in X^{\prime \prime}$ or $k \in Y \backslash X$ and $p^{\prime \prime} \Vdash_{\mathbb{Q}_{\mu+1}} " k \notin X^{\prime \prime}$ so long as the name $X^{\prime}$ is still a $\mathbb{Q}_{\mu+1}$ name; in other words, the antichains in $\mathbb{Q}_{\mu}$ deciding membership in $X^{\prime}$ remain maximal in $\mathbb{Q}_{\mu+1}$.

This last equivalence is guaranteed by the choice of $W_{\mu}$, because the relevant dense sets are definable from $X^{\prime}$, which belongs to $\mathfrak{M}_{\mu}$, and $\mathbb{Q}_{\mu}$. The claim now follows from the definition of $\Vdash_{*}$. 
It will be now be shown that it is possible to choose $V_{\mu} \subseteq W_{\mu}$ such that for each $p \in \mathbb{Q}_{\mu}$ and $\mathbb{Q}_{\mu}$-name, $Y \in \mathfrak{M}_{\mu}$, for a subset of $\omega$

$$
p \cup 1_{V_{\mu}} \cup 0_{W_{\mu} \backslash V_{\mu}} \Vdash_{\mathbb{Q}_{\mu+1}} " \Phi^{*}\left(W_{\mu}\right) \cap Y \not \#^{*} \Phi^{*}\left(V_{\mu}\right) "
$$

If this is not possible then it follows from Claim 1 that there is no $V_{\mu}$ such that $V_{\mu} \subseteq W_{\mu}$ and such that for each $p \in \mathbb{Q}_{\mu}$ and each $\mathbb{Q}_{\mu}$-name for a subset of $\omega$, $Y \in \mathfrak{M}_{\mu}$

$$
\left(p, 1_{V_{\mu}} \cup 0_{W_{\mu} \backslash V_{\mu}}\right) \Vdash_{*} \cdot " \partial_{\mathfrak{F}} \Phi\left(\left[W_{\mu}\right]\right) \cap[Y] \not \#^{*} \partial_{\mathfrak{F}} \Phi\left(\left[V_{\mu}\right]\right) "
$$

It will be shown that this implies that there is are continuous functions $\Psi_{n}$, for $n \in \omega$, such that for each $C \subset W_{\mu}$ there is $n \in \omega$ such that $\Psi_{n}(C) \equiv^{*} \Phi^{*}(C)$, thus contradicting the fact that $\mathfrak{M}$ is being assumed to satisfy the conclusion of Lemma 2.6.

Here is how to conclude this. For each $p \in \mathbb{Q}_{\mu}$ and $A \subset W_{\mu}$ define $q_{p}(A)=$ $\left(p, 1_{A} \cup 0_{W_{\mu} \backslash A}\right)$ and $\bar{q}_{p}(A)=p \cup 1_{A} \cup 0_{W_{\mu} \backslash A}$. Given $p$ and $r$ in $\mathbb{Q}_{\mu}, n \in \omega$ and $Y \in \mathfrak{M}_{\mu}$, a $\mathbb{Q}_{\mu}$-name for a subset of $\omega$, define $\psi_{p, r, n, Y}(A)$ to be the set

$$
\left\{k \in \Phi^{*}\left(W_{\mu}\right) \backslash n:\left(\forall p^{\prime}\right)\left(p^{\prime} \cup p \cup \overline{q_{r}}(A) \text { is not a function or } p^{\prime} \Vdash_{\mathbb{Q}_{\mu}} " k \in Y^{\prime}\right\}\right.
$$

for $A \in \operatorname{dom}\left(\psi_{p, r, n, Y}\right)=\left\{A \subset W_{\mu}: \bar{q}_{r}(A) \cup p\right.$ is a function $\}$.

It will first be shown that for each $A \subset W_{\mu}$ there are $p, r, n$ and $Y$ such that $\Phi^{*}(A) \equiv^{*} \psi_{p, r, n, Y}(A)$. To see see this recall that there is some $r_{A} \in \mathbb{Q}_{\mu}$ and some $Y_{A} \in \mathfrak{M}_{\mu}$, a $\mathbb{Q}_{\mu}$-name for a subset of $\omega$, such that

$$
q_{r_{A}}(A) \Vdash_{*} " \Phi^{*}\left(W_{\mu}\right) \cap Y_{A} \not \equiv^{*} \Phi^{*}(A) "
$$


fails to be true and $q_{r_{A}}(A)$ is a function. Hence, there is some $p_{A} \in \mathbb{Q}_{\mu}$ such that $p_{A} \cup \bar{q}_{r_{A}}(A)$ is a function and there is some $n_{A} \in \omega$ such that for each $p^{\prime}$ and $k \geq n_{A}$ either $p^{\prime} \cup p_{A} \cup \bar{q}_{r_{A}}(A)$ is not a function or $p^{\prime} \| \mathbb{Q}_{\mu} " k \in\left(\Phi^{*}\left(W_{\mu}\right) \cap\right.$ $\left.Y_{A}\right) \Delta\left(\Phi^{*}(A)\right)^{\prime}$.

If $k \in \psi_{p_{A}, r_{A}, n_{A}, Y_{A}}(A) \backslash \Phi^{*}(A)$ and $k>n_{A}$ then the definition of $\psi_{p_{A}, r_{A}, n_{A}, Y_{A}}(A)$ implies that $p^{\prime} \Vdash_{\mathbb{Q}_{\mu}} " k \in Y_{A}$ " whenever $p^{\prime}$ is such that $p^{\prime} \cup p_{A} \cup \bar{q}_{r_{A}}(A)$ is a function. But this means that $p^{\prime} \Vdash_{\mathbb{Q}_{\mu}} " k \in\left(\Phi^{*}\left(W_{\mu}\right) \cap Y_{A}\right) \Delta\left(\Phi^{*}(A)\right)$ " contradicting the choices of $p_{A}, r_{A}, n_{A}$ and $Y_{A}$.

On the other hand, suppose that $k$ belongs to $\left(\Phi^{*}(A) \cap \Phi^{*}\left(W_{\mu}\right)\right) \backslash n_{A}$. If $k \notin \psi_{p_{A}, r_{A}, n_{A}, Y_{A}}(A)$ then there exists $p^{\prime}$ such that $p^{\prime} \cup p_{A} \cup \bar{q}_{r_{A}}(A)$ is a function and $p^{\prime} \|_{\mathbb{Q}_{\mu}} " k \in Y_{A} "$. It follows that there is $p^{\prime \prime} \supset p^{\prime}$ such that $p^{\prime \prime} \Vdash_{\mathbb{Q}_{\mu}} " k \notin Y_{A} "$. If $p^{\prime \prime} \cup p_{A} \cup \bar{q}_{r_{A}}(A)$ is a function then $p^{\prime \prime} \Vdash_{\mathbb{Q}_{\mu}} " k \in\left(\Phi^{*}\left(W_{\mu}\right) \cap Y_{A}\right) \Delta\left(\Phi^{*}(A)\right)$ " once again contradicting the choice of $p_{A}, r_{A}, n_{A}$ and $Y_{A}$. But why should $p^{\prime \prime} \cup p_{A} \cup \bar{q}_{r_{A}}(A)$ be a function?

The fact that it is possible to choose $p^{\prime \prime}$ such that $p^{\prime \prime} \cup p_{A} \cup \bar{q}_{r_{A}}(A)$ is a function follows from the choice of $W_{\mu}$. Recall that $W_{\mu}$ was chosen so that $W_{\mu} \in \mathcal{O}$ for every open set $\mathcal{O} \subseteq \mathcal{P}\left(\mathcal{W}_{\mu}^{\prime}\right)$ which is definable from $\mathfrak{M}_{\mu}$ and $\mathbb{Q}_{\mu}$. Moreover, the set $\mathcal{O}$ consisting of all $W \subseteq W_{\mu}^{\prime}$ such that there exists $e \in[W]^{<\aleph_{0}}$ such that for all $\epsilon: e \rightarrow 2$ there exists $p_{\epsilon}$ satisfying one of the following three conditions

- $\epsilon \cup p_{A} \cup r_{A}$ is not a function

- $p_{\epsilon} \Vdash_{\mathbb{Q}_{\mu}} " k \notin Y_{A} "$ and $p_{\epsilon} \cup p_{A} \cup r_{A} \cup \epsilon$ is a function and $p_{\epsilon}\lceil W=\epsilon$ 
- $\epsilon \cup p_{A} \cup r_{A} \Vdash_{\mathbb{Q}_{\mu}} " k \in Y_{A} "$

is easily seen to be dense and open in $W_{\mu}^{\prime}$ and to be definable from $\mathbb{Q}_{\mu}$ and $Y_{A}, p_{A}$ and $r_{A}$ - all of which belong to $\mathfrak{M}_{\mu}$. Hence $W_{\mu}$ belongs to this dense open set $\mathcal{O}$. Now let $e \in\left[W_{\mu}\right]^{<\aleph_{0}}$ witness this fact; in other words, letting $\epsilon=\left(p_{A} \cup r_{A} \cup 1_{A} \cup 0_{W_{\mu} \backslash A}\right)\left\lceil e\right.$, there is some $p_{\epsilon}$ such that

- $p_{\epsilon} \Vdash_{\mathbb{Q}_{\mu}} " k \notin Y_{A} "$

- $p_{\epsilon} \cup p_{A} \cup r_{A}$ is a function

- $p_{\epsilon}\left\lceil W_{\mu}=p_{A} \cup r_{A} \cup 1_{A} \cup 0_{W_{\mu} \backslash A}\right)\lceil e$

because the other two alternatives are not possible in light of the fact that $k \notin \psi_{p_{A}, r_{A}, n_{A}, Y_{A}}(A)$ and $A \in \operatorname{dom}\left(\psi_{p_{A}, r_{A}, n_{A}, Y_{A}}\right)$. It follows that setting $p^{\prime \prime}=p_{\epsilon}$ yields the desired condition.

Now observe that the functions $\psi_{p, r, n, Y}$ are all Borel. This contradiction to Lemma 2.6 finishes the proof of the theorem.

\section{$3 \quad$ Remarks and Open Questions}

It is worth noting that not only has it been shown that it is consistent that there is a nontrivial automorphism yet all automorphisms are somewhere trivial, but also that this is consistent with $\mathrm{MA}_{\omega_{1}}$. Combining the arguments of this paper 
with those of [6] it is possible to show that that it is consistent (even with $\left.\mathrm{MA}_{\omega_{1}}\right)$ that every autohomeomorphism of $\beta \mathbb{N} \backslash \mathbb{N}$ is somewhere trivial while any two P-points have the same topological type in the sense that there is an autohomeomorphism of $\beta \mathbb{N} \backslash \mathbb{N}$ mapping one to the other. In this model it will of course follow that there are $2^{\mathfrak{c}}$ autohomeomorphisms of $\beta \mathbb{N} \backslash \mathbb{N}$ which raises the following question.

Question 3.1 Is it consistent that there are only $2^{\aleph_{0}}$ automorphisms of $\mathcal{P}(\omega) /[\omega]<\aleph_{0}$ but that there is, nevertheless, a nontrivial automorphism?

The nontrivial automorphism constructed with Velickovic's order is much more than somewhere trivial - the collection of subsets of $\omega$ where it is trivial forms a maximal ideal. Given any automorphism $\Phi: \mathcal{P}(\omega) /[\omega]<\aleph_{0} \rightarrow$ $\mathcal{P}(\omega) /[\omega]^{<\aleph_{0}}$ define $\mathcal{J}(\oplus)$ to be the collection of sets on which $\Phi$ is trivial. It is not difficult to check that $\mathcal{J}(\oplus)$ is always an ideal but it is not clear what else can be said about it.

Question 3.2 Does $M A_{\omega_{1}}$ imply that $\mathcal{J}(\oplus) \neq[\omega]^{<\aleph, ~ f o r ~ e a c h ~ a u t m o r p h i s m ~} \Phi$ of $\mathcal{P}(\omega) /[\omega]<\aleph_{0}$ ?

Question 3.3 Does $M A_{\omega_{1}}$ imply that $\mathcal{J}(\oplus)$ is the intersection of maximal ideals for every automorphism $\Phi$ of $\mathcal{P}(\omega) /[\omega]<\aleph_{0}$ ?

\section{References}


1. U. Abraham, M. Rubin, and S. Shelah, On the consistency of some partition theorems for continuous colorings and the structure of $\aleph_{1}$-dense real order types, Annals of Pure and Applied Logic 29 (1985), 123-206.

2. K. P. Hart and J. van Mill, Open problem on $\beta \omega$, Open Problems in Topology (G. M. Reed and J. van Mill, eds.), North-Holland, pp. 99-125.

3. W. Just, A modification of Shelah's oracle-c.c. with applications, Transactions of the American Mathematical Society ??? (1989), ??-??

4. S. Shelah, Proper forcing, Lecture Notes in Mathematics, vol. 940, SpringerVerlag, Berlin, 1982.

5. S. Shelah and J. Steprāns, PFA implies all automorphism are trivial, Proc. Amer. Math. Soc. 104 (1988), 1220-1225.

6. J. Steprāns, Martin's Axiom and the transitivity of P-points, Submitted to Israel Journal of Mathematics, 19.

7. B. Velickovic, Definable automorphisms of $\mathcal{P}(\omega) /\{\rangle \backslash$, Proc. Amer. Math. Soc 96 (1986), 130-135.

8. B. Velickovic, OCA and automorphisms of $\mathcal{P}(\omega) /\{\rangle \backslash$, preprint, 1990. 